\title{
Zur Kenntnis der Thymusnucleinsäuren.
}

\author{
III. Mitteilung. ${ }^{1}$ ) \\ Von \\ H. Stendel.
}

(Aus dem physiologischen Institut zu Heidelberg.)

(Der Redaktion zugegangen am 2. Oktober 1905.)

Bei der Spaltung der Nucleinsäuren durch siedende Säuren entstehen unter anderen Zersetzungsprodukten Körper sowohl aus der Purin- wie aus der Pyrimidingruppe. Beide Körperklassen stehen chemisch in enger Beziehung, weil man sowohl Glieder der Puringruppe aus Pyrimidinderivaten aufbauen als auch Purinderivate zu Pyrimidinverbindungen abbauen kann; auch physiologisch muß eine nahe Verwandtschaft beider Körperklassen, z. B. bei der Entstehung der Harnsäure im Tierorganismus, vorausgesetzt werden. Nachdem also das Vorkommen von Pyrimidinkörpern unter den hydrolytischen Zersetzungsprodukten der Nucleinsäuren festgestellt war, als das Thymin als Pyrimidinderivat charakterisiert war und sich ihm kurz darauf noch das Cytosin und das Uracil hinzu gesellten, lag nichts näher, als auch hier an einen Zusammenhang zwischen Purin- und Pyrimidinverbindungen in der Weise $\mathrm{zu}$ denken, daß die Körper des einfachen Pyrimidinringes oder wenigstens ein Teil von ihnen einer Einwirkung der siedenden Säuren auf die Purinbasen ihre Entstehung verdanken.

Aber einer solchen Annahme standen doch manche Bedenken gegenüber: so ließ sich erstens das Thymin von keinem bekannten Purinderivat ableiten, seine Entstehung aus einem Purinkern wird auch durch seine Konstitutionsformel wenig

1) I. Mitteilung, Diese Zeitschrift, Bd. XLII, S. 165.

II. Mitteilung, Diese Zeitschrift, Bd. XLIII, S. 402. 
wahrscheinlich gemacht; ferner wurde in einem besonderen Versuch untersucht, ob aus Guanin bei der Einwirkung siedender Schwefelsäure Pyrimidinderivate entständen, allein auch hier ließen sich keine solche Verbindungen isolieren. Endlich sprachen die Versuche, in denen eine quantitative Bestimmung der Zersetzungsprodukte angestrebt wurde, die bei der Einwirkung siedender Säuren auf Nucleinsäure entstehen, nicht für die Auffassung, daß die Purinbasen Muttersubstanzen der Pyrimidinkörper seien. Wurde mit hydrolysierenden Säuren unter Zugabe starker Reduktionsmittel gespalten, so wurde gefunden, daß z. B. Jodwasserstoffsäure und Phosphor einen beträchtlichen Teil des Guanins, Salzsäure und Zinnchlorür einen großen Teil des Guanins und Adenins zerstörte; eine entsprechende Vermehrung der Pyrimidinfraktionen ließ sich aber nicht feststellen, hier wurden in sämtlichen Versuchen nahezu die gleichen Zahlen erhalten, der Anteil der zerstörten Purinbasen hatte keinen Einfluß auf die Größe des Betrages der Pyrimidinderivate.

Im weiteren Verfolg dieser Frage habe ich nun einen Versuch ausgeführt, in dem die Nucleinsäure so energisch mit Schwefelsäure gespalten wurde, daß sämtliche Purinbasen zerstört wurden; es ist dies leicht auszuführen. ${ }^{1}$ ) Man sollte denken, daß wenigstens unter solchen Bedingungen sich ein Einfluß auf die Quantität der Pyrimidinverbindungen, die gegen siedende Säuren resistenter wie die Purinbasen sind, zeige; aber auch hier hat der Versuch zugunsten der Ansicht entschieden, daß die Pyrimidinderivate unabhängig von den Purinbasen aus dem Molekül der Nucleinsäure bei der Säurespaltung hervorgehen.

Als Ausgangsmaterial dienten wie bei meinen früheren Untersuchungen $100 \mathrm{~g}$ lufttrockenes a-nucleinsaures Kupfer aus Thymus. Die Reaktionsflüssigkeit wurde auf $1000 \mathrm{ccm}$ aufgefüllt, dann eine Bestimmung des Gesamtstickstoffs gemacht.

$5 \mathrm{ccm}$, nach Kjeldahl verascht, sättigen $38,8 \mathrm{ccm} \mathrm{n/s0-0xalsäure}$ $=0,05422 \mathrm{~g} \mathrm{~N}=10,844 \%$.

$5 \mathrm{ccm}$, nach $\mathrm{Kjeldahl}$ verascht, sättigen $38,5 \mathrm{ccm}$ n/10-0xalsäure $=0,05390 \mathrm{~g} \mathrm{~N}=10,780 \%$.

1) Kossel und Neumann, Ber., Bd. XXVII, S. 2215. 
Der Gesamtstickstoff in der Zer'setzungsflüssigkeit betrug also 10,812 g N (Durchschnittszahl).

Nach Entfernung des Kupfers mit Schwefelwasserstoff, der Schwefelsäure mit Barythydrat und des Ammoniaks durch Kochen mit Baryumcarbonat wurde die Flüssigkeit auf 5 Volumprozent Schwefelsäure gebracht und mit Phosphorwolframsäure ausgefällt. Bei der Aufteilung des Phosphorwolframsäureniederschlages mit Silbernitrat und Baryt konnten keine Purinbasen erhalten werden, es fiel nur Cytosinsilber, das mit Salzsäure vom Silber befreit und mit Natriumpikrat ausgefällt wurde. Erhalten wurden 6,95 g. (Nach einmaligem Umkristallisieren F. P. 270 ${ }^{\circ}$ ) Aus dem Filtrat der Phosphorwolframsäurefällung wurden in bekannter Weise 4,992 $\mathrm{g}$ Thymin erhalten.

Nun enthalten $6,95 \mathrm{~g}$ Cytosinpikrat 2,25 g Cytosin mit $0,853 \mathrm{~g} \mathrm{~N}$, $4,992 \mathrm{~g}$ Thymin entsprechen $1,110 \mathrm{~g} \mathrm{~N}$, oder, in Prozenten des Gesamtstickstoffs ausgedrückt:

$$
\begin{aligned}
& \text { Cytosin- } \mathrm{N}=7,9 \% \\
& \text { Thymin- } \mathrm{N}=10,3 \% .
\end{aligned}
$$

(Es sei zu diesen Zahlen noch bemerkt, daß 81\% des Stickstoffs wiedergefunden wurden, der in der Flüssigkeit vorhanden war, in der die Fällung mit Phosphorwolframsäure vorgenommen wurde.)

Vergleicht man diese Zahlen mit den Resultaten, die ich früher erhalten habe, ${ }^{1}$ ) so ergibt sich für die in Frage kommenden Werte folgende Übersicht:

\begin{tabular}{c|c|c|c|c}
\hline $\begin{array}{c}\text { In Prozenten } \\
\text { des Gesamt- } \\
\text { stickstoffs } \\
\text { gefunden } \\
\text { für }\end{array}$ & $\begin{array}{c}\text { Jodwasser- } \\
\text { stoffsäure } \\
\text { und } \\
\text { Phosphor }\end{array}$ & $\begin{array}{c}\text { Salzsäure } \\
\text { und } \\
\text { Zinnchlorür }\end{array}$ & $\begin{array}{c}\text { Schwefel- } \\
\text { säure, } \\
\text { gewöhnliche } \\
\text { Spaltung }\end{array}$ & $\begin{array}{c}\text { Schwefel- } \\
\text { säure, } \\
\text { tiefe } \\
\text { Spaltung }\end{array}$ \\
\hline Guanin .... & 3,61 & 3,15 & 10,07 & 0 \\
Adenin .... & 13,45 & 4,76 & 16,39 & 0 \\
Cytosin . . . & 11,45 & 10,15 & 11,47 & 7,9 \\
Thymin . . . & 15,88 & 11,91 & 13,11 & 10,3
\end{tabular}

1) Diese Zeitschrift, Bd. LXIII, S. 404. 
Die Menge der Pyrimidinverbindungen hat sich also nicht, einer Abnahme der Purinkörper entsprechend, vermehrt und man müßte jetzt schon zu ganz gekünstelten Erklärungsversuchen greifen, wollte man noch länger behaupten, daß die Pyrimidinderivate, die man bei Säurespaltung findet, weiter nichts wie Zersetzungsprodukte der Purinbasen seien. ${ }^{1}$ ) Mir will es auch scheinen, als ob man eine solche Erklärung ihrer Herkunft häufig theoretischen Erwägungen über eine eventuelle Struktur der Nucleinsäure zuliebe herangezogen habe.

Die bisher bekannten Elementaranalysen der Nucleinsäuren haben zur Aufstellung einer Formel geführt, die von Schmiedeberg ${ }^{2}$ ) als $\mathrm{C}_{40} \mathrm{H}_{56} \mathrm{~N}_{14} \mathrm{O}_{26} \mathrm{P}_{4}$ angegeben wird. Für ein Kupfersalz würde daraus $\mathrm{C}_{40} \mathrm{H}_{58} \mathrm{Cu}_{2} \mathrm{~N}_{14} \mathrm{O}_{26} \mathrm{P}_{4}$ sich ergeben. Auch die von mir am nucleinsauren Kupfer ausgeführten Analysen stimmen einigermaßen zu einer solchen Formel, trotzdem ich mein Material auf ganz anderem Wege wie Schmiedeberg gewonnen habe. Nur die N-Werte sind durchweg zu hoch.

Die Substanz wurde bei $120^{\circ}$ bis zur Gewichtskonstanz getrocknet. Sie bräunt sich dabei nicht, nur die grüne Farbe wird dem Wasserverluste entsprechend blasser und heller; dann wurde das nucleinsaure Kupfer mit Soda und Salpeter verbrannt, das Kupfer als Sulfür und der Phosphor als Magnesiumpyrophosphat gewogen. Der Stickstoff wurde nach Kjeldahl bestimmt.

$$
\begin{aligned}
& 0,2420 \mathrm{~g}=0,0296 \mathrm{~g} \mathrm{Cu}_{2} \mathrm{~S}=9,77 \% \mathrm{Cu} \\
& 0,2642 \text {, }=0,0300 \text {, , }=9,07 \% \text {, } \\
& =0,0842, \mathrm{Mg}_{8} \mathrm{P}_{2} \mathrm{O}_{7}=8,87 \% \mathrm{P} \\
& 0,4986 \text {, }=0,1574, \quad, \quad=8,79 \% \text {, }
\end{aligned}
$$

$0,2358 \mathrm{~g}$ verbrauchen $24,2 \mathrm{ccm}$ n/10-0xalsäure $=14,37 \% \mathrm{~N}$

0,1200 > > 12,4 , $=14,47 \%$,

0,1344 > > 13,9 > $=14,48 \%$,

Berechnet:

$\begin{array}{lrrr}\mathrm{Cu}=9,10 \% & 9,77 \% & 9,07 \% & \\ \mathrm{P}=8,89 \% & 8,87 \% & 8,79 \% & \\ \mathrm{~N}=14,10 \% & 14,37 \% & 14,47 \% & 14,48 \%\end{array}$

1) Selbstverständlich soll damit nichts über die physiologische Beziehung beider Gruppen ausgesagt sein.

2) Arch. f. exp. Path. u. Pharm., Bd. XLIII, S. 57. 
336 H. Steudel, Zur Kenntnis der Thymusnucleinsäuren. III.

Aber die Unterbringung der Spaltungsprodukte in diese Formel macht große Schwierigkeiten. Rechnet man nur je ein Molekül Guanin, Adenin, Thymin und Cytosin als Spaltungsprodukte, eine Annahme, die natürlich ganz hypothetisch ist, so erhält man schon 15 Stickstoffatome, während die Formel nur auf $14 \mathrm{~N}$ berechnet ist. Ebenso gibt eine Berechnung der Mengen der einzelnen Spaltungskörper, falls sie in molekularen Mengen aus der Formel für die Nucleinsäure hervorgehen sollen, Zahlen, die nicht mit den experimentellen Ergebnissen in Einklang zu bringen sind. Die Mitteilung solcher Berechnungen entbehrt einer Berechtigung, bis die quantitative Aufteilung weiter fortgeschritten sein wird.

Die Untersuchungen werden von mir fortgesetzt werden. 